\title{
Exponential Operator for Bearing Estimation
}

\author{
Youssef $\mathrm{Khmou}^{1}$, Said Safi ${ }^{1}$ and Miloud Frikel ${ }^{2}$ \\ ${ }^{1}$ Department of Mathematics and informatics \\ Polydisciplinary faculty, Beni Mellal, Morocco \\ ${ }^{2}$ GREYC laboratory, ENSICAEN School, Caen University, France \\ ${ }^{1}$ khmou.y@gmail.com,safi.said@gmail.com, ${ }^{2}$ mfrikel@greyc.ensicaen.fr
}

\begin{abstract}
In this paper, we present an original spectral technique for estimating the Direction of Arrival (DoA) of narrowband sources impinging on an array of sensors. In this approach, we use the exponential of inter spectral matrix of received data with Gaussian function to annihilate the eigenvalues corresponding to the signal subspace in order to obtain an approximation of the projector into the noise subspace, this mechanism requires the knowledge of the minimum eigenvalue which we compute using the power method.

We elaborate this new concept with some tools of quantum formalism and we confirm the accuracy of its theoretical basis by Monte Carlo simulation results comparatively to the standard high resolution techniques.
\end{abstract}

Keywords: Exponential Operator, spectral matrix, DoA, subspace, Gaussian, angular spectrum, localization

\section{Introduction}

In spectral analysis, localization of radiating sources is an active field of research [1], its applications span several domains including, but not limited to, Geolocation systems [2], submarine acoustics [3], seismology [4] and astronomy [5]. The well known techniques of estimating the Directions Of arrivals (DOA) rely on the spectral decomposition [6], QR and LU factorizations [7] and Singular Value Decomposition (SVD) [8] of the received data or the corresponding spectral matrix.

The initial research started with the beamforming [9], this concept is based on the principle of interferometry [10] which has a disadvantage of low resolution capability, in other words if we have few number of sensors, the beamforming cannot separate multiple sources if they lie in the main lobe of the array. Several improvements have been made to increase the resolution power such as Burg's Maximum Entropy method [11], Capon beamformer [12] and linear prediction [13]. in the 80th a revolutionary high resolution technique based on eigendecomposition was derived by Schmidt [6], since that time, many works extended the idea of spectral decomposition such as ESPRIT [8] and Minimum Norm [11] methods, however the computational complexity of these contributions were high. In searching for fast solutions, new concepts were proposed, namely the Propagator $[14,15]$ and the Ermolaev-Gershman [16] operators.

Recently the ongoing researches are rooted in statistical physics, to enhance the resolution power of the existing DOA spectra, such as the compressive sensing concept [17], and the consistency of the spectra [18] when the dimensions of the system tend to infinity.

In the future, statistical signal processing will rely on the invention of new architecture of computers and trans-receiving systems precisely the quantum devices [19]. Indeed, the quantum theory is based on probability density functions for system measurement. In this paper, we introduce an original technique based on matrix exponential of the covariance matrix of the received data to compute the angular spectrum for locating the radiating 
sources, the mechanism introduced is efficient in annihilating the signal subspace in the covariance matrix. In the next section, we present the mathematical model to describe the received signals by the antenna, in the third section we elaborate our formalism and finally we confirm the validity of the proposed method by simulation results.

\section{Signal Model}

We consider a wavefield, consisting of superposition of $P$ narrowband sources, that is received by an array of $N$ equally spaced and identical sensors, the received signals can be described in the frequency domain by the following equation:

$$
X(f)=A(\theta, f) s(f)+n(f)
$$

$X(f) \in \mathbb{C}^{N \times 1}$ is the state vector at the frequency $f, s(f)=\left[s_{1}(f), \ldots, s_{P}(f)\right]^{T}$ is the waveform vector and $A(\theta, f) \in \mathbb{C}^{N \times P}$ is Vandermonde steering matrix of the array $A(\theta, f)=\left[a_{1}(\theta, f), \ldots, a_{P}(\theta, f)\right]$, the $i^{t h}$ steering vector is given by :

$$
a\left(\theta_{i}\right)=\frac{1}{\sqrt{N}}\left[1, e^{-j \varphi_{i}}, \ldots, e^{-j(N-1) \varphi_{i}}\right]
$$

Where the phase $\varphi_{i}=2 \pi \lambda^{-1} d \sin \theta_{i}$, the $i^{\text {th }}$ Direction Of Arrival (DoA) is $\theta_{i}, d$ is the inter-element distance and $\lambda$ is the wavelength of incoming radiations. Any element of the steering matrix $a_{i j}$ can be written as the following:

$$
a_{i j}=e^{-\vec{k}_{i} \cdot \vec{r}_{j}}
$$

$\vec{k}_{i}$ is the wave vector of $i^{\text {th }}$ source and $\vec{r}_{j}$ is the position of the $j^{\text {th }}$ sensor $\vec{r}_{j}=(j-$ 1) $\vec{e}_{x}$.

$n(f)=\left[n_{1}(f), \ldots, n_{N}(f)\right]^{T}$ is the additive zero mean complex random process with flat spectrum, which is added to model the perturbations originating from multiple sources ( thermal noise, scattering, reflections,...), the corresponding joint probability density function is given by :

$$
p(n(t))=\frac{1}{\pi^{N}\left|\Gamma_{n}\right|} \exp \left\{\frac{-n^{+}(t) \Gamma_{n}^{-1} n(t)}{2}\right\}
$$

$(.)^{+}$denotes the conjugate transpose operator, the matrix $\Gamma_{n}$ is defined by the equation:

$$
\Gamma_{n}=\lim _{K \rightarrow+\infty} \frac{1}{K} \sum_{t=1}^{K} n(t) n^{+}(t)=\sigma^{2} I_{N}
$$

This equation indicates that the noise in uncorrelated between the sensors with the same power $\sigma$ on each sensor, and statistically independent of the waveforms $\mathrm{s}(\mathrm{t})$. The inter spectral matrix $\Gamma$ is computed from finite number of samples $K$, its theoretical expression is given by the following equation:

$$
\Gamma=\lim _{K \rightarrow+\infty} \frac{1}{K} \sum_{t=1}^{K} X(t) X^{+}(t)=A \Gamma_{S S} A^{+}+\Gamma_{n}=\Gamma_{S}+\Gamma_{n}
$$

$\Gamma_{S S} \in \mathbb{C}^{P \times P}$ is the correlation matrix of waveforms, if we assume that the sources are statistically independent, the correlation matrix is written as $\Gamma_{S S}=\delta_{i j} \sigma_{i}^{2}$ with $\sigma_{i}$ being the power of the $i^{\text {th }}$ source. For the rest of the paper, we introduce the braket notation, for any vector $|e\rangle \in \mathbb{C}^{N \times 1},|e\rangle^{+}=\langle e|$, the high resolution techniques are based the spectral theorem, the eigendecomposition is given by : 


$$
\Gamma=\sum_{\mathrm{i}=1}^{\mathrm{N}} \lambda_{\mathrm{i}}\left|u_{i}\right\rangle\left\langle u_{i}\right|=U \Lambda U^{+}=U_{s} \Lambda_{s} U_{s}^{+}+U_{n} \Lambda_{n} U_{n}^{+}
$$

$U_{s} \in \mathbb{C}^{N \times P}$ is the signal subspace and $U_{n}=U_{s}^{\perp}$ where the complete base is obtained by the relation $U_{s} U_{s}^{+}+U_{n} U_{n}^{+}=I_{N}, \lambda_{\mathrm{i}}$ is the $i^{t h}$ eigenvalue that corresponds to the eigenvector $\left|u_{i}\right\rangle$ with norm of unity $\|\left|u_{i}\right\rangle \|_{2}=1$ :

$$
\Gamma\left|u_{i}\right\rangle=\lambda_{\mathrm{i}}\left|u_{i}\right\rangle
$$

$U$ is unitary operator which can rewritten using the resolution of the identity :

$$
\sum_{\mathrm{i}=1}^{\mathrm{N}}\left|u_{i}\right\rangle\left\langle u_{i}\right|=\mathrm{I}_{\mathrm{N}}
$$

An approximate value of noise power can be obtained by:

$$
\hat{\sigma}^{2}=\frac{1}{N-P} \sum_{i=P+1}^{N} \lambda_{\mathrm{i}}
$$

For given steering vector $\left|a\left(\theta_{i}\right)\right\rangle$, the one dimensional localization function is calculated by the function $f\left(\theta_{i}\right)=\left\langle a\left(\theta_{i}\right)\left|U_{n} U_{n}^{+}\right| a\left(\theta_{i}\right)\right\rangle$, this metric is null if $\theta_{i}$ is the true Direction of Arrival (DoA) [6], with $P_{n}=U_{n} U_{n}^{+}$is the projector into the noise subspace, its discrete spectrum is given by:

$$
\sigma_{P_{n}}=\left[0_{1 \times P} \mid 1_{1 \times N-P}\right]
$$

One of the fast techniques to obtain an approximation of the projector $P_{n}$ is the Ermolaev and Gershman operator [16], given by the following equation:

$$
P_{n}=\lim _{m \rightarrow+\infty}\left(\left(\frac{\Gamma}{\lambda_{t h}}\right)^{m}+I_{N}\right)^{-1}
$$

The parameter $\lambda_{t h}$ is the threshold between the smallest signal eigenvalue $\lambda_{P}$ and the largest noise eigenvalue $\lambda_{P+1}$, generally the parameter $m$ is set to 10 . In this next section we present a new mechanism to construct the operator $P_{n}$ using matrix exponential.

\section{Exponential Operator}

Given the self adjoint operator $\Gamma$, a matrix function $f(\Gamma)$ is applied to its spectrum $f(\Gamma)=\operatorname{Uf}(\Lambda) U^{+}$, generally the eigenvalues of $\Gamma$ are given in the following order:

$$
\sigma_{\Gamma}=\left\{\lambda_{1} \geq \lambda_{2} \geq \cdots \geq \lambda_{P}>\lambda_{P+1} \cong \cdots \cong \lambda_{N}=\sigma^{2}\right\}
$$

In order to normalize the noise eigenvalues $\lambda_{q}(q=P+1, \ldots, N)$ while annihilating the signal eigenvalues $\lambda_{r}(r=1, \ldots, P)$, we apply the Gaussian function described by the following equation :

$$
f(\lambda)=e^{-\beta\left(\lambda-\lambda_{\text {Min }}\right)^{2}}
$$

This concept is illustrated in Figure 1, the half width at half maximum is $\sqrt{\log (2) / \beta}$. 


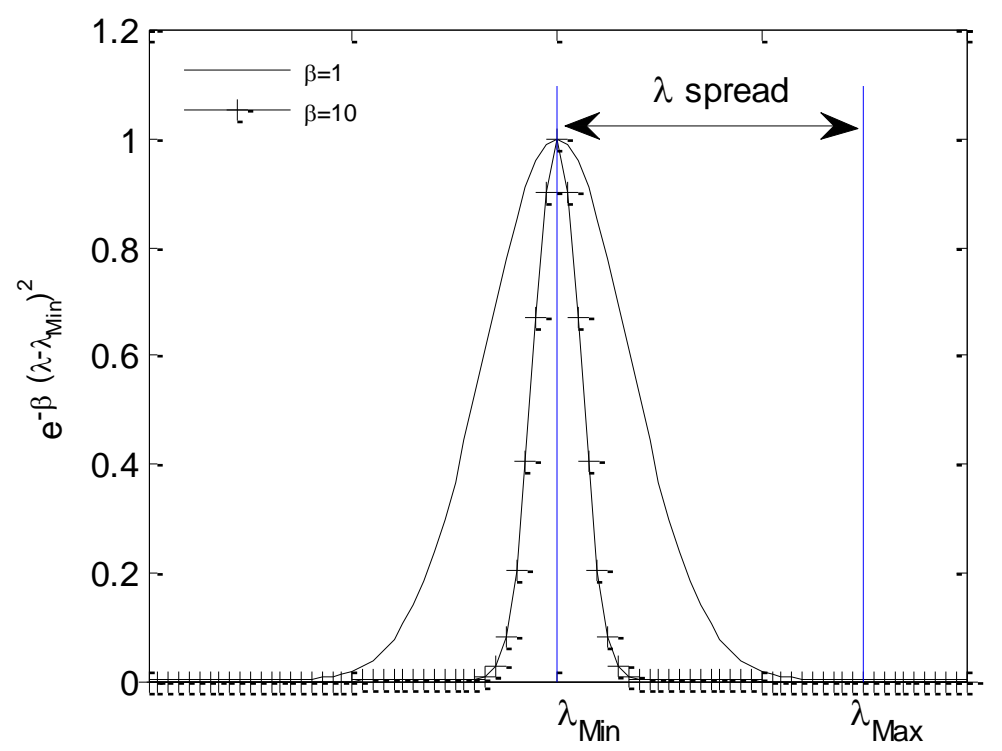

Figure 1. Gaussian Function Applied to $\sigma_{\Gamma}$ with Parameters $\left(\beta, \lambda_{M i n}\right)$

The knowledge of the minimum eigenvalue is required, many techniques can be implemented to find an approximate value, in this paper we use the power method [20] to calculate the largest eigenvalue $\lambda_{\operatorname{Max}}$ from which we find $\lambda_{\operatorname{Min}}$ using the condition number:

$$
\tau(\Gamma)=\frac{\lambda_{\operatorname{Max}}}{\lambda_{\operatorname{Min}}}
$$

The power method is initialized by choosing a random vector $|\Phi\rangle$ such that $\||\Phi\rangle \|_{\infty}=1$, for iteration $m \geq 2$ we perform the following calculations:

$$
\begin{gathered}
\left|\Phi_{m+1}\right\rangle=\Gamma\left|\Phi_{m}\right\rangle \\
\mu_{m}=\left\langle\Phi_{m} \mid \Phi_{m+1}\right\rangle \\
\left|\Phi_{m}\right\rangle=\frac{\left|\Phi_{m+1}\right\rangle}{\left\langle\Phi_{m} \mid \Phi_{m+1}\right\rangle}
\end{gathered}
$$

After few iterations, $\mu_{m}$ tends to $\lambda_{\operatorname{Max}}$, the smallest eigenvalue is given by the following relationship:

$$
\lambda_{\text {Min }}=\frac{\lambda_{\operatorname{Max}}}{\tau(\Gamma)}=\frac{\lambda_{\text {Max }}}{\|\Gamma\|_{2}\left\|\Gamma^{-1}\right\|_{2}}
$$

The second parameter that we need to adjust is the width $\beta$ of the function in equation (12), the parameter $\beta$ depends on the spread of the signal eigenvalues, however we do not give any theoretical guidelines for modeling its value, but we propose the value $\beta=10$ which corresponds to half width of $\sqrt{\log (2) / \beta} \cong 0.26$ which is narrow enough to not let any signal eigenvalue $\lambda_{q}$ to be selected. The straightforward application of the above concept to the spectral matrix yields to:

$$
e^{-\beta\left(\Gamma-\lambda_{\text {Min }} I_{N}\right)^{2}}=I_{N}+\sum_{k=1}^{+\infty} \frac{-\beta^{k}\left(\Gamma-\lambda_{\operatorname{Min}} I_{N}\right)^{2 k}}{k !}
$$


The serie in the above equation always converges, the spectral matrix is written as a sum of two self adjoint operators $\Gamma=\Gamma_{\mathrm{s}}+\Gamma_{\mathrm{n}}$, we can remark that the two operators commute:

$$
\left[\Gamma_{\mathrm{s}}, \Gamma_{\mathrm{n}}\right]=\Gamma_{\mathrm{s}} \Gamma_{\mathrm{n}}-\Gamma_{\mathrm{n}} \Gamma_{\mathrm{s}}=0_{\mathrm{N}}
$$

Therefore the square of spectral matrix is written as $\Gamma^{2}=\Gamma_{s}^{2}+\Gamma_{n}^{2}+2 \Gamma_{\mathrm{s}} \Gamma_{\mathrm{n}}$ where the operators verify the Gloden Thompson inequality [21]:

$$
\operatorname{Tr}\left\{e^{\Gamma_{\mathrm{s}}+\Gamma_{\mathrm{n}}}\right\} \leq \operatorname{Tr}\left\{e^{\Gamma_{\mathrm{s}}} e^{\Gamma_{\mathrm{n}}}\right\}
$$

Using equations (18) and (19), we give the following proposition to compute an approximation of $P_{n}$.

Proposition 3.1: The operator $e^{-\beta\left(\Gamma-\lambda_{M i n} I_{N}\right)^{2}}$ is an approximation to the noise projector $P_{n}=U_{n} U_{n}^{+}$

$$
\begin{gathered}
e^{-\beta\left(\Gamma-\lambda_{\text {Min }} I_{N}\right)^{2}}=e^{-\beta \Gamma^{2}} e^{2 \beta \lambda_{\text {Min }} \Gamma} e^{-\beta \lambda_{\text {Min }}^{2} I_{N}} \\
=e^{-\beta \Gamma_{S}^{2}} e^{-2 \beta \Gamma_{\mathrm{s}} \Gamma_{\mathrm{n}}} e^{-\beta \Gamma_{n}^{2}} e^{2 \beta \lambda_{\text {Min }} \Gamma_{s}} e^{2 \beta \lambda_{\text {Min }} \Gamma_{n}} e^{-\beta \lambda_{\text {Min }}^{2} I_{N}} \\
\cong U_{n} U_{n}^{+}
\end{gathered}
$$

Proof: Using the spectral decomposition we have:

$$
\begin{gathered}
e^{-\beta\left(\Gamma-\lambda_{\text {Min }} I_{N}\right)^{2}}=U e^{-\beta\left(\Lambda-\lambda_{\text {Min }} I_{N}\right)^{2}} U^{+}=\sum_{i=1}^{N} e^{-\beta\left(\lambda_{i}-\lambda_{\text {Min }}\right)^{2}}\left|u_{i}\right\rangle\left\langle u_{i}\right| \\
=\sum_{q=1}^{P} e^{-\beta\left(\lambda_{q}-\lambda_{\text {Min }}\right)^{2}}\left|u_{q}\right\rangle\left\langle u_{q}\left|+\sum_{r=P+1}^{N} e^{-\beta\left(\lambda_{r}-\lambda_{\text {Min }}\right)^{2}}\right| u_{r}\right\rangle\left\langle u_{r}\right| \\
\cong \sum_{r=P+1}^{N}\left|u_{r}\right\rangle\left\langle u_{r}\right|=U_{n} U_{n}^{+}=P_{n}
\end{gathered}
$$

From the computational viewpoint, the equation (21) can be calculated using different ways such the approximation theory or the linear differential equations, indeed it was explained in [22] that there exist nineteen techniques to compute the matrix exponential. Since the minimum eigenvalue represents the noise power, we can write the following equation:

$$
\lim _{S N R \rightarrow+\infty} P_{n}=e^{-\beta \Gamma^{2}}
$$

For given normalized state vector $\left|a\left(\theta_{i}\right)\right\rangle$ in Hilbert space, the angular spectrum is obtained by calculating the inverse of the expectation value of the proposed operator $\left\langle P_{n}\right\rangle_{\theta_{i}}^{-1}$, using the inverse of Rayleigh quotient, the localization function is given by the equation :

$$
f\left(\theta_{i}\right)=\left\langle P_{n}\right\rangle_{\theta_{i}}^{-1}=\frac{\left\langle a\left(\theta_{i}\right) \mid a\left(\theta_{i}\right)\right\rangle}{\left\langle a\left(\theta_{i}\right)\left|P_{n}\right| a\left(\theta_{i}\right)\right\rangle}=\frac{1}{\left\langle a\left(\theta_{i}\right)\left|e^{-\beta\left(\Gamma-\lambda_{\text {Min }} I_{N}\right)^{2}}\right| a\left(\theta_{i}\right)\right\rangle}
$$

In the next section, we present some simulation results to confirm the validity of the proposed operator, that we compare with several high resolution spectral techniques.

\section{Simulation Results:}

We consider an array, consisting of $N=11$ omnidirectional and identical sensors, that is intercepting wavefield of $P=4$ punctual and narrowband sources impinging 
from directions $\theta=\left[-40^{\circ}, 5^{\circ}, 30^{\circ}, 35^{\circ}\right]$, the signals are considered to be zero mean complex random processes $s_{i}(t) \sim \mathcal{C N} \mathcal{N}(0,1 \mathrm{watt})$ and statistically independent $\Gamma_{s s}=$ $\delta_{i j} \sigma_{i}^{2}$. The carrier frequency is $f_{c}=1 \mathrm{GHz}$ which corresponds to interelement distance of $d=15 \mathrm{~cm}$ which corresponds to an array aperture of $L_{\lambda}=1.5 \mathrm{~m}$. The number of measurements is $K=200$ samples. In the first experiment we set $S N R=5 d B(S N R=$ $20 \log _{10}\left(1 / \sigma^{2}\right)$ ). Figure 2 represents an average of $L=100$ Monte Carlo runs of equation (24), the obtained spectrum has a high resolution property with no side lobes.

In the second test, we study the variation of the eigenvalues of the operator in equation (18) as a function of degree of perturbation $\sigma_{P_{n}}=f(S N R)$, the results are presented in Figure 3. We realize that when the perturbation is high $(S N R<0), \sigma_{P_{n}}$ contains at least the minimum eigenvalue which belongs to the noise subspace, when the $S N R$ increases starting from $0 d B$, we can observe that all the noise eigenvalues are normalized while the signal eigenvalues are always null.

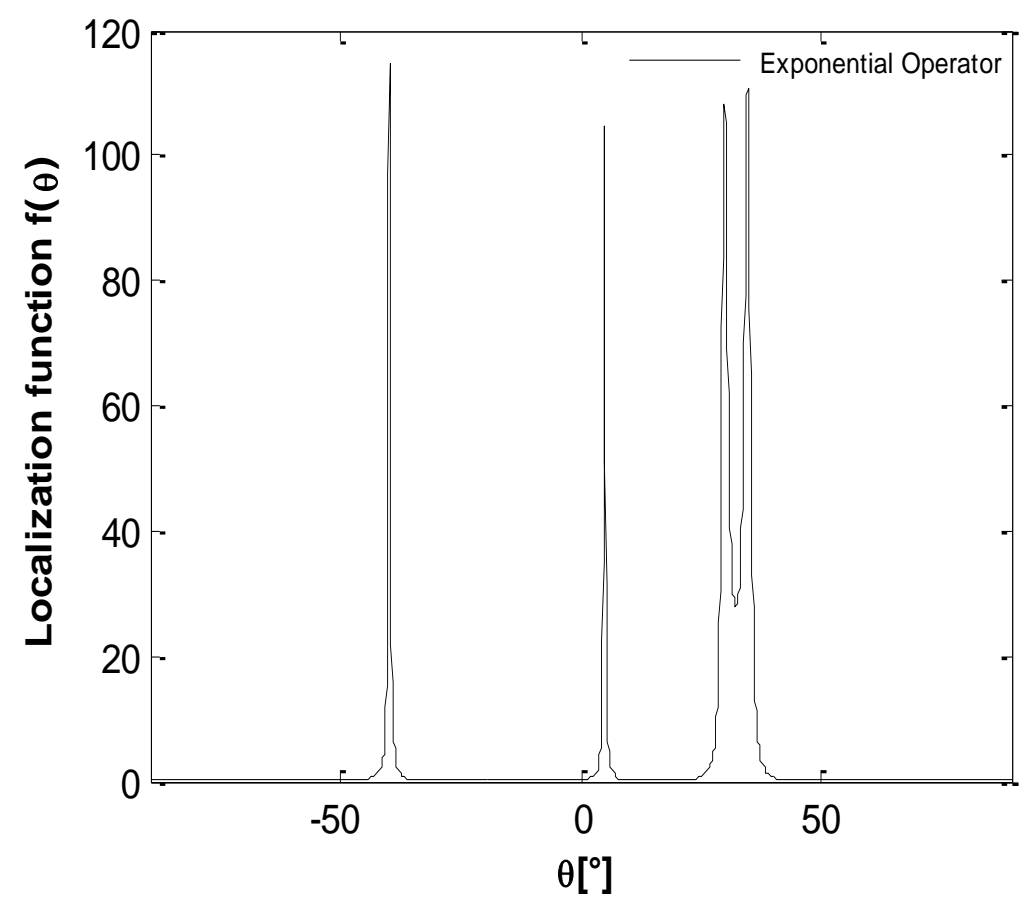

Figure 2. Average of $L=100$ Realizations with $N=11, P=4, \theta=$ $\left[-40^{\circ}, 5^{\circ}, 30^{\circ}, 35^{\circ}\right], K=200, d=\lambda / 2$ and $S N R=5 d B$ 


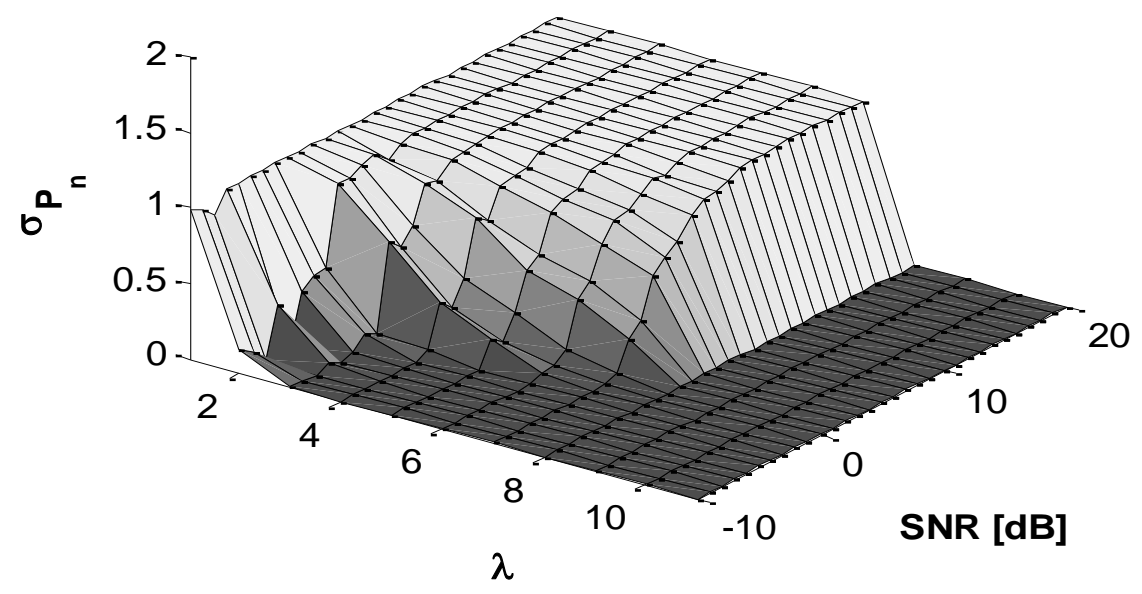

Figure 3. $\sigma_{P_{n}}=f(S N R)$ with $N=11, P=4, \theta=\left[-40^{\circ}, 5^{\circ}, 30^{\circ}, 35^{\circ}\right], K=200$ and $d=\lambda / 2$

In the third test we compare the performance of the localization function with four different spectra which are Ermolaev and Gershman operator [16], Multiple Signal Classification techniques [6], Minimum Variance Distortionless Response operator [12] and standard beamforming [9] using the same conditions as in the first test, Figure 4 represents the comparison in $d B$ scale, the proposed operator, Ermolaev and Gershman operator with parameter $m=10$, and Schmidt subspace (MUSIC) are all equivalent in these conditions.

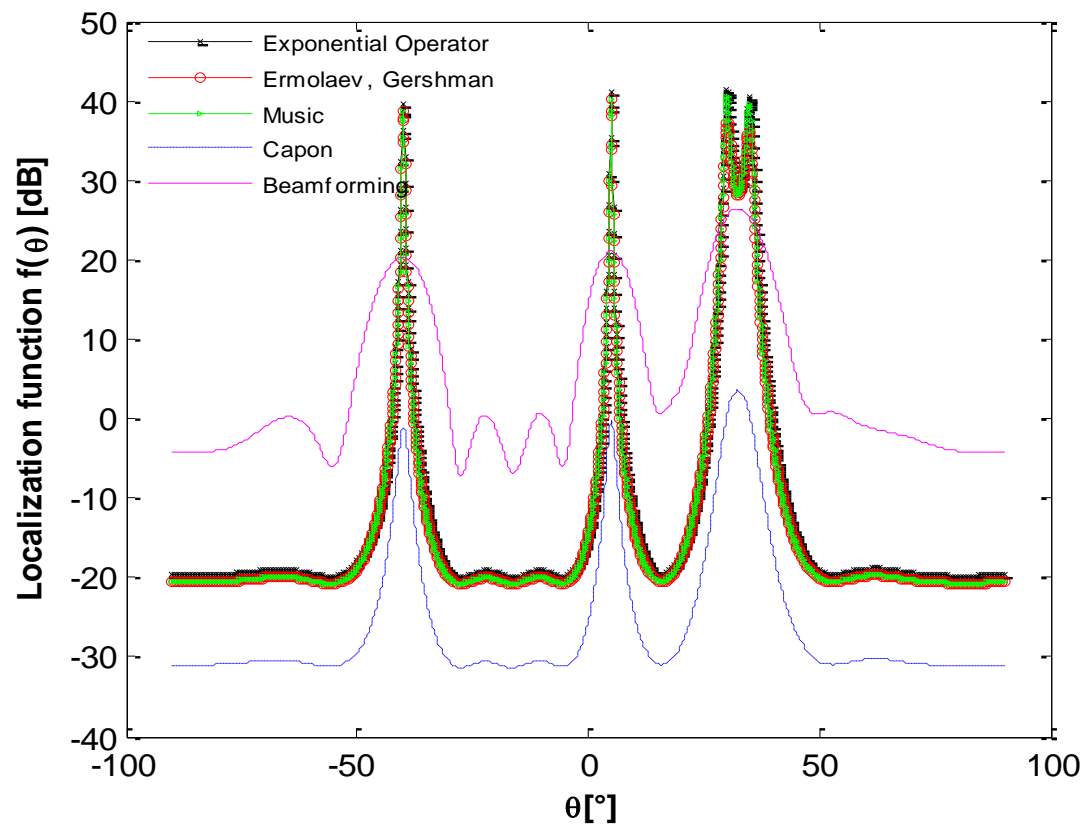

Figure 4. Average of $L=100$ Realizations of Proposed Operator with Four Different Spectra with $N=11, P=4, \theta=\left[-40^{\circ}, 5^{\circ}, 30^{\circ}, 35^{\circ}\right], K=200$, $d=\lambda / 2$ and $S N R=5 d B$ 
To generalize this result, we compare the Root Mean Square Error (RMSE) of the four high resolution spectra with varying $S N R$, the results are presented in Figure 5. The obtained functions prove that the proposed operator has the same performance, in these conditions, of the same high resolution spectra starting from $S N R=0 \mathrm{~dB}$.

\section{Conclusion}

We have introduced, in this paper, an original spectral technique for computing the angular spectrum of the narrowband sources whose radiations are received by an array of sensors. New formalism is presented, based on quantum theory, to calculate an approximation of the projector into the noise subspace using Gaussian function of the spectral matrix, this new mechanism requires only the knowledge of the minimum eigenvalue. The simulation results proved that the proposed operator has the same performance with the existing high resolution spectra.

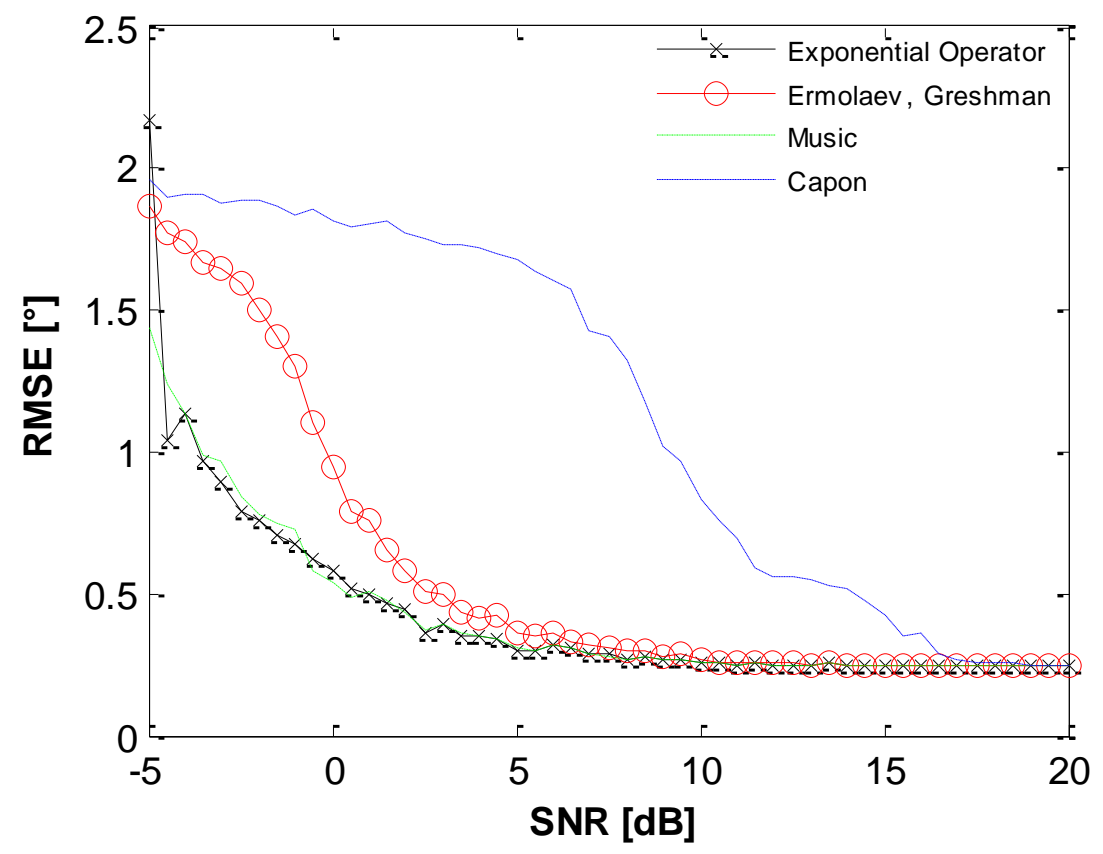

Figure 5. Average of $L=100$ Realizations of RMSEs for each Value of SNR with $N=11, P=4, \theta=\left[-40^{\circ}, 5^{\circ}, 30^{\circ}, 35^{\circ}\right], K=200$ and $d=\lambda / 2$.

\section{References}

[1] H. Krim and M. Viberg, "Two Decades of Array Signal Processing Research”, IEEES Signal Processing Magazine, vol. 13, (1996), pp. 67-94.

[2] M. Frikel, B. Targui, S. Safi and M. M'Saad, "Bearing detection of noised wideband sources for geolocation", Control \\& Automation (MED), 2010 18th Mediterranean Conference, (2010) June 23-25, pp. 1650,1653 .

[3] T. Li and A. Nehorai, "Maximum Likelihood Direction-of-Arrival Estimation of Underwater Acoustic Signals Containing Sinusoidal and Random Components", Signal Processing, IEEE Transactions, vol. 59, no. 11, (2011) November, pp. 5302,5314.

[4] T.-Y. Chang and H.-L. Cui, "Determination of Direction of Arrival of Seismic Wave by a Single Triaxial Fiber Optic Geophone", Defence Technology, vol. 9, no. 1, (2013) March, pp. 1-9, ISSN 22149147.

[5] L. Chen, A. Aminaei, H. Falcke and L. Gurvits, "Optimized estimation of the Direction of Arrival with single tripole antenna", Antennas and Propagation Conference (LAPC), 2010 Loughborough, (2010) November 8-9, pp. 93-96.

[6] R. O. Schimdt, "Multiple Emitter Location and Signal Parameter Estimation”, IEEE Trans. On Antennas Propagation, vol. 34, no. 3, (1986) March, pp. 276-280. 
[7] J. Marot, C. Fossati and S. Bourennane, "Fast subspace-based source localization methods", Sensor Array and Multichannel Signal Processing Workshop, 2008. SAM 2008. 5th IEEE, (2008) July 21-23, pp. 203-206.

[8] R. Roy and T. Kailath, "ESPRIT -Estimation of Signal Parameters Via Rotational Invariance Techniques", IEEE Trans. On Acoust., Speech, Signal Processing, vol. 37, no. 7, (1989) July, pp. 984995.

[9] M. S. Bartlett, "Periodogram analysis and Continuous spectra", Biometrika, vol. 37, no. 1-2, (1950), pp. $1-16$.

[10] Z. W. Barber, C. Harrington, C. W. Thiel, W. R. Babbitt and R. Krishna Mohan, "Angle of arrival estimation using spectral interferometry", Journal of Luminescence, ISSN 0022-2313, vol. 130, no. 9, (2010) September, pp. 1614-1618.

[11] L. C. Godara, "Application of antenna arrays to mobile communications, II. Beam-forming and direction-of-arrival considerations", Proceedings of the IEEE, vol. 85, no. 8, (1997) August, pp. 11951245 .

[12] J. Capon, "High-resolution frequency-wavenumber spectrum analysis", Proceedings of the IEEE, vol. 57, no. 8, (1969) August, pp. 1408-1418.

[13] J. Makhoul, "Linear prediction: A tutorial review", Proceedings of the IEEE, vol. 63, no. 4, (1975) April, pp. 561-580.

[14] S. Marcos, A. Marsal and M. Benidir, "The Propagator Method for Source Bearing Estimation”, Signal Processing, vol. 42, no. 2, (1995), pp. 121-138.

[15] S. Marcos and M. Benidir, "On A High Resolution Array Processing Method Non-Based on the Eigenanalysis Approach”, Acoustics, Speech, and Signal Processing, 1990. ICASSP-90., International Conference (1990).

[16] V. T. Ermolaev and A. B. Gershman, "Fast algorithm for minimum-norm direction-of-arrival estimation", Signal Processing, IEEE Transactions, vol. 42, no. 9, (1994) September, pp. 2389-2394.

[17] F. Roemer, M. Ibrahim, R. Alieiev, M. Landmann, R. Thomae, S. Galdo and G. Del, "Polarimetric Compressive Sensing Based DOA Estimation", Smart Antennas (WSA), 2014 18th International ITG Workshop, (2014) March 12-13, pp. 1-8.

[18] X. Mestre and M.-A. Lagunas, "Modified Subspace Algorithms for DoA Estimation With Large Arrays", Signal Processing, IEEE Transactions, vol. 56, no. 2, (2008) February, pp. 598-614.

[19] I. Djordjevic, "Quantum Information Processing and Quantum Error Correction", Academic Press, ISBN : 9780123854919.

[20] M. Frikel and S. Bourennane, "High-resolution methods without eigendecomposition for locating the acoustic sources", Applied Acoustics, vol. 52, no. 2, (1997) October, pp. 139-154, ISSN 0003-682X.

[21] S. Golden, "Lower bounds for the Helmholtz function", Phys. Rev. (2), 137:B1127-B1128,1965.

[22] C. Moler and C. van Loan, SIAM Rev., vol. 45, no. 3, (2003).

\section{Authors}

Youssef Khmou obtained the B.Sc. degree in Physics and M.Sc. degree in computer science from polydisciplinary faculty, in 2010 and from Faculty of Science and Technics Beni Mellal, Morocco, in 2012, respectively. Now he is Ph.D student and his research interests include statistical signal and array processing and statistical physics.

Said Safi received the B.Sc.degree in Physics (option Electronics) from Cadi Ayyad University, Marrakech, Morocco in 1995, M.Sc. and Doctorate degrees from Chouaib Doukkali University and Cadi Ayyad Univerrsity, in 1997 and 2002, respectively. He has been a Professor of information theory and telecommunication systems at the National School for applied Sciences, Tangier, Morocco, from 2003 to 2005. Since 2006, he is a Professor of applied mathematics and programming at the Faculty of Science and Technics, Beni Mellal, Morocco. In 2008 he received the Ph.D. degree in Telecommunication and Informatics from the Cadi Ayyad University. His general interests span the areas of communications and signal processing, estimation, time-series analysis, and system identification - subjects on which he has published 14 journal papers and more than 60 conference papers. Current research topics focus on transmitter and receiver diversity techniques for single- and multi-user fading communication channels, and wide-band wireless communication systems

Miloud Frikel received his $\mathrm{Ph} . \mathrm{D}$. degree from the center of mathematics and scientific computation CNRS URA 2053, France, in array processing. Currently, he is with the 
GREYC laboratory (CNRS URA 6072) and the ENSICAEN as Assistant Professor. From 1998 to 2003, Dr. Frikel was with the Signal Processing Lab, Institute for Systems and Robotics, Institute Superior Tecnico, Lisbon, as a researcher in the field of wireless location and statistical array processing, after been a research engineer in a software company in Munich, Germany. He worked in the Institute for Circuit and Signal Processing of the Technical University of Munich. His research interests span several areas, including statistical signal and array processing, cellular geolocation (wireless location), spacetime coding, direction finding and source localization, blind channel identification for wireless communication systems, and MC-CDMA systems. 